\title{
Decline in ovarian reserve may be an undiagnosed reason for unexplained infertility: a cohort study
}

Burak Yücel ${ }^{1}$, Sefa Kelekci², Emine Demirel ${ }^{2}$

${ }^{1}$ Department of Gynecology and Obstetrics, Kanuni Sultan Suleyman Research and Training Hospital, Istanbul, Turkey

2Department of Gynecology and Obstetrics, Katip Celebi University Faculty

of Medicine, Ataturk Research and Training Hospital, Izmir, Turkey

Submitted: 11 November 2015

Accepted: 22 February 2016

Arch Med Sci 2018; 14, 3: 527-531

DOI: https://doi.org/10.5114/aoms.2016.58843

Copyright $\odot 2016$ Termedia \& Banach

\section{Abstract}

Introduction: Unexplained infertility refers to the absence of a definable cause for a couple's failure to achieve pregnancy. Reproductive aging plays a role in pathogenesis of unexplained infertility. We investigated the results of ovarian reserve tests in unexplained infertility.

Material and methods: The patients were divided into two groups: unexplained infertility $(n=148)$ and male factor infertility $(n=112)$. Follicle-stimulating hormone, estradiol, inhibin b levels and anti-Müllerian hormone levels were evaluated. Antral follicle count and ovarian volume measurements were performed.

Results: The demographic variables were comparable. Follicle-stimulating hormone levels were higher in the unexplained infertility group than the male factor infertility group, although this difference did not reach statistical significance $(p=0.071)$. Estradiol levels, inhibin b concentrations and ovarian volume showed no difference between groups. However, antral follicle count was significantly lower in the unexplained infertility group than the male factor infertility group $(p=0.023)$. The median anti-Müllerian hormone concentrations were significantly lower in the unexplained infertility group 1.42 $(0.4-6.2)$ than in the male factor infertility group $(2.04(0.64-8.2) ; p=0.001)$. Conclusions: Although anti-Müllerian hormone values and antral follicle count were higher than the low thresholds, a statistically significant decline of ovarian reserve in the unexplained infertility group was found in the present study. This might be an undiagnosed reason for unexplained infertility.

Key words: ovarian reserve, infertility, anti-Müllerian hormone, unexplained infertility.

\section{Introduction}

Unexplained infertility (UEI) is a diagnosis of exclusion after the basic infertility evaluation reveals normal semen parameters, evidence of ovulation, patent fallopian tubes, and no other obvious cause of infertility [1].

Two potential explanations can be described for UEl: there is a specific cause, but not one that can be identified with existing diagnostic tests. On the other hand, there is truly no abnormality, but the couple's natural fertility is at the extreme lower end of the normal range, possibly due to advanced reproductive aging. The higher incidence of UEI in women over 35 years is also evidence for the role of reproductive aging in the pathogenesis. In addition, there is enormous variability in ovarian

\author{
Corresponding author: \\ Burak Yücel MD \\ Department of Gynecology \\ and Obstetrics \\ Kanuni Sultan \\ Suleyman Research \\ and Training Hospital \\ Seyit Gazi M. \\ MKP Bulvari, No. 1 \\ 38100 Istanbul, Turkey \\ Phone: +90 5326009554 \\ E-mail: \\ drburakyucel@gmail.com
}


reserve at similar ages and even young patients can already have a diminished ovarian reserve. Diminished ovarian reserve could be an important factor when no obvious explanation for the failure of pregnancy to occur is found.

Ovarian reserve generally describes the size and quality of the remaining ovarian follicular pool. Studies of the mechanisms involved in reproductive aging and its clinical consequences have stimulated efforts to measure ovarian reserve. The most appropriate ovarian reserve screening tests to use in practice are basal follicle-stimulating hormone (FSH), estradiol, inhibin $\mathrm{B}$, antral follicle count (AFC), ovarian volume and anti-Müllerian hormone $(\mathrm{AMH})[2,3]$.

In the literature, only a few studies have explored the association between ovarian reserve and UEI $[4,5]$. The suggestion of diminished ovarian reserve has been observed in concentrations of hormones of the pituitary-ovarian axis in women with UEI compared with a control group [6]. In this study, we aimed to investigate the role of undetectable reproductive aging by using the ovarian reserve test in the etiology of UEI.

\section{Material and methods}

The study was carried out in accordance with the Declaration of Helsinki for medical research involving human subjects and approved by the local ethical committee (Protocol Number 2011-12), and a written informed consent form was obtained from all patients.

\section{Patient selection}

The study was a prospective cohort study. All women were referred from an gynecology outpatient clinic to the infertility department for further examination and treatment. The women were healthy except for their infertility and did not use any hormonal medication. Infertility was determined by standard infertility evaluation based on the guidelines of American Society for Reproductive Medicine [7]. It includes assessment of ovulation, hormone analyses, a hysterosalpingogram, at least two semen analyses and, if indicated, laparoscopy.

From all couples, a fertility history and a diagnostic work-up were obtained. Duration of infertility had to be more than 12 months. Assessment of ovulation was performed by measuring the serum progesterone levels on days 21-22 of the menstrual cycle. If the serum progesterone levels were higher than $10 \mathrm{ng} / \mathrm{ml}$, ovulation was confirmed. In view of our study aims, having elevated FSH concentration (> $10 \mathrm{IU}$ ) was an explanatory category for UEI. In our practice, women with elevated FSH concentration are diagnosed as having premature ovarian failure, not UEI. Older age (such as > 40) was not an exclusion criteria for this study. Measurement of FSH concentration was the unique ovarian reserve test at the first step. Tubal passage was demonstrated by hysterosalpingogram. Transvaginal ultrasonography was performed to detect unsuspected ovarian or uterine pathology. If necessary, laparoscopy or hysteroscopy was performed to exclude factors such as endometriosis or intrauterine pathologies. Male factor infertility (MFI) was defined as a couple with otherwise UEI in whom semen analysis did not meet the standard for normality as defined by the World Health Organization in 2010. In this study, to avoid selection bias, we selected the control group among patients with $\mathrm{MFI}$, not from the completely fertile population.

\section{Ovarian reserve tests}

Further ovarian reserve tests were performed in the fertility department. Venous blood samples were taken from the antecubital regions of all patients between 8:00 a.m. and 9:00 a.m. in the early follicular phase (days 2-4) of the menstrual cycle. Serum samples were stored at $-20^{\circ} \mathrm{C}$ and assayed for estradiol $\left(E_{2}\right)$. Ovarian volume and the total numbers of antral follicles measuring 2-10 mm in diameter were evaluated by the same operator blinded to patient information in the same morning. Ovarian volume was calculated with the equation of an ellipsoid (length $\times$ width $\times$ thickness $\times$ $0.523)$. Anti-Müllerian hormone concentration was measured using the enzyme immunoassay AntiMüllerian Hormone ELISA kit (reference A11893) provided by Immunotech (Beckman Coulter, Marseille, France). A 7.5 MHz, transvaginal probe (Medison SonoAce X8 Ultrasound, Samsung Medison, Seoul, Korea) was used in all examinations.

\section{Statistical analysis}

Statistical analyses were performed using SPSS (Statistical Package for the Social Sciences) software (SPSS 19.0 for Windows; SPSS Inc. Chicago, IL). The Kolmogorov-Smirnov test of normality was used to evaluate the distributions. Non-normally distributed metric variables were analyzed by Mann-Whitney U-test. Qualitative data were compared using Fisher's exact test. Multiple logistic regression analysis was performed in order to find the significant results of the ovarian reserve tests associated with UEI while controlling for age. Statistical significance was considered as $p<0.05$.

\section{Results}

The groups consisted of 148 women with UEI and 112 women with MFI. The characteristics of the study population are depicted in Table I. The 
patients with UEI were somewhat older than the patients with $\mathrm{MFI}$, although the difference was not statistically significant. Duration of infertility, ratio of primer infertility and body mass index values were similar in both groups.

The results of the ovarian reserve tests are given in Table II. Follicle-stimulating hormone levels were higher in the UEl group than the MFI group, although this difference did not reach statistical significance $(p=0.071)$. There were no differences between the two groups with regard to estradiol, inhibin B levels and ovarian volumes. Antral follicle count was significantly lower in the UEI group than the MFI group ( $p=0.023)$. The median AMH concentrations were significantly lower in the UEI group $1.42(0.4-6.2)$ than in the MFI group (2.04 $(0.64-8.2) ; p=0.001)$.

Multiple logistic regression analysis was performed for the significant results of ovarian reserve tests between women with UEI and controls (Table III). The data demonstrated that age was an independent risk factor for UEl. The entire model was statistically significant $(p<0.001)$. While adjusting for age, $\mathrm{AMH}$ was found to be statistically significant $(p=0.012)$, but AFC was not $(p=0.354)$. After controlling for confounders, unexplained infertility was associated with a decrease of $\mathrm{AMH}(\mathrm{OR}=0.433,95 \% \mathrm{Cl}: 0.226-0.830)$.

\section{Discussion}

The ovarian reserve tests under this study $\mathrm{FSH}$, estradiol, inhibin $\mathrm{B}, \mathrm{AFC}$ and $\mathrm{AMH}$ - were analyzed in the UEI group and the MFI group, which was considered as the control group. Although $\mathrm{FSH}$, estradiol and inhibin levels were similar in both groups, women with UEI had lower AFC and AMH levels in this study.

Couples with UEI are thought to represent a group of patients with a defect in fecundity; however, that defect is not detected by the standard infertility evaluation [1]. Couples with UEI demonstrate both diminished and delayed fecundity compared to fertile women. In a review, Guzick et al. [8] determined the cycle fecundity rate with expectant management in couples with UEI to be $1.3-4.1 \%$, compared to the generally accept-

Table I. Characteristics of the study groups

\begin{tabular}{|lccc|}
\hline Parameter & Unexplained infertility group & Male factor infertility group & $P$-value \\
\hline Number of patients & 148 & 112 & \\
\hline Age & $33(25-42)$ & $32(29-44)$ & 0.701 \\
\hline Duration of infertility & $2(1.2-5)$ & $2(1.1-8)$ & 0.591 \\
\hline Primer infertility & $80(54.05 \%)$ & $68(60.71 \%)$ & 0.289 \\
\hline Body mass index & $24(18.01-34.02)$ & $24(19-32.27)$ & 0.531 \\
\hline
\end{tabular}

Table II. Results of the ovarian reserve tests in the study groups

\begin{tabular}{|lccc|}
\hline Parameter & Unexplained infertility group & Male factor infertility group & $P$-value \\
\hline FSH [IU/l] & $7.52(4.21-9.88)$ & $6.96(5.1-9.37)$ & 0.071 \\
\hline Estradiol $[\mathrm{pg} / \mathrm{ml}]$ & $51.50(27-86)$ & $43.50(25-71)$ & 0.108 \\
\hline Inhibin B [pg/ml] & $119(40-145)$ & $120(52-150)$ & 0.298 \\
\hline Ovarian volume $[\mathrm{ml}]$ & $6.2(3.2-10.96)$ & $6.06(3.3-12.2)$ & 0.634 \\
\hline Antral follicle count, $n$ & $9(3-16)$ & $10(3-23)$ & 0.023 \\
\hline AMH $[\mathrm{ng} / \mathrm{ml}]$ & $1.42(0.4-6.2)$ & $2.04(0.64-8.2)$ & 0.001 \\
\hline
\end{tabular}

Table III. Multiple logistic regression model for the results of the ovarian reserve tests

\begin{tabular}{|c|c|c|c|c|c|c|}
\hline \multirow[t]{2}{*}{ Parameter } & \multirow[t]{2}{*}{$\beta$} & \multirow[t]{2}{*}{ S.E. } & \multirow[t]{2}{*}{$P$-value } & \multirow[t]{2}{*}{ OR } & \multicolumn{2}{|c|}{$95 \% \mathrm{Cl}$ for OR } \\
\hline & & & & & Lower & Upper \\
\hline Constant & 6.327 & 1.762 & $<0.001$ & 559.545 & - & - \\
\hline Age & -0.156 & 0.046 & $<0.001$ & 0.856 & 0.782 & 0.936 \\
\hline Antral follicle count, $n$ & 0.094 & 0.101 & 0.354 & 1.099 & 0.901 & 1.340 \\
\hline $\mathrm{AMH}[\mathrm{ng} / \mathrm{ml}]$ & -0.837 & 0.332 & 0.012 & 0.433 & 0.226 & 0.830 \\
\hline
\end{tabular}


ed cycle fecundity of $20 \%$ in proven fertile couples. When we exclude false-negative results of standard tests for infertility, there are two potential explanations for UEl: First, there is a specific cause, but not one that can be identified with existing diagnostic tests. Second, more importantly, there truly is abnormality, and the couple's natural fertility is at the extreme lower end of the normal age, possibly due to advanced reproductive aging [2].

The basal FSH concentration has been recognized as the simplest test to measure ovarian reserve [9]. Follicle-stimulating hormone levels can vary significantly. Follicle-stimulating hormone levels greater than $10 \mathrm{IU} / \mathrm{l}$ have high specificity for diminished ovarian reserve. In the present study, FSH levels were higher in the UEI group than the MFI group, although this difference did not reach statistical significance $(p=0.071)$. A single measurement of estradiol concentration has little value to show ovarian reserve, but can provide additional information that helps in the interpretation of the basal FSH level. Inhibin B is generally not regarded as a reliable measure of ovarian reserve. Ovarian volume decreases with follicular depletion. The measurement has high inter-cycle and inter-observer variability, so ovarian volume has very limited clinical utility as an ovarian reserve test [6]. Estradiol and inhibin B levels and ovarian volumes were similar in both groups in our study.

Antral follicle count is proportional to the number of primordial follicles remaining. It is an indirect but useful measure of ovarian reserve [10] Antral follicle count was lower in the UEI group in the present study. The decline was statistically significant. Antral follicles are described as follicles that reach a stage of development where they are responsive to $\mathrm{FSH}$, which stimulates and supports more advanced stages of development. The decline in AFC in the UEI group may provide an undetectable defect in early development of the follicle.

Anti-Müllerian hormone is an autocrine and paracrine regulator that is produced by the granulosa cells of preantral and small antral follicles. Its levels are gonadotropin-independent and exhibit little variation within and between cycles. Thus, $\mathrm{AMH}$ is a very promising screening test for diminished ovarian reserve (DOR), especially in women at high risk for DOR $[11,12]$. Anti-Müllerian hormone levels were significantly lower in the UEI group in this study.

Van Rooij et al. [4] studied the predictive potential of ovarian reserve tests with regard to the occurrence of an ongoing pregnancy in UEI patients. When the results of this study were evaluated, the ovarian reserve tests were not correlated with each other. Apart from normal values of FSH, high AFC such as 14 follicles and high inhibin $B$ and estradiol levels, median AMH levels were $1.1 \mathrm{ng} / \mathrm{ml}$ in the mild semen factor group and 1.0 for UEI. These values were near to low threshold values $(0.2-0.7 \mathrm{ng} / \mathrm{ml})$ of $\mathrm{AMH}$ that are generally accepted. This limitation could be caused by patient selection of the authors. Older age $(>40)$ or having elevated FSH concentrations (> $15 \mathrm{IU} / \mathrm{l}$ ) was not considered as an explanatory category by the authors. As a result, some women who should be diagnosed with DOR were included in the study population as UEl cases. Murto et al. [5] studied hormone levels and determined the predictive value for long-term possibility of live birth in women in a UEI group and an MFI group. Inhibin B values were significantly low in this study. Median values of inhibin were $37.1 \mathrm{pg} / \mathrm{ml}$ for the UEl group and 47.5 for MFI. These values were under the globally accepted low threshold for inhibin B. However, the other ovarian reserve test did not support this finding. The whole study population was small (71 infertile women) in this study. Hence, when divided into subgroups (42 for UEI and 29 for MFI), data would be inadequate to confirm differences.

Unexplained infertility likely represents the lower extreme of the normal distribution of reproductive efficiency that cannot be detected reliably by standard methods. Currently, there is no uniformly accepted definition of DOR. The ideal ovarian reserve test should yield consistent results and be highly specific. Basal FSH is the most commonly used ovarian reserve test, but AFC and $\mathrm{AMH}$ are promising predictors with significant potential advantages [2]. In our study, FSH and other ovarian reserve tests were similar for the UEI group and controls. However, AFC and $\mathrm{AMH}$ values are significantly lower in the UEI group. In support of liberal application of the ovarian reserve test, more specific and sensitive tests, such as $\mathrm{AMH}$, do not apply to all subfertile women [7]. Consequently, some women with DOR are diagnosed with UEl.

In conclusion, ovarian reserve tests have become a routine element of diagnostic evaluation for subfertility [2]. It is recommended testing in women at increased risk of having a DOR such as UEl. Although AMH values and antral follicle count were higher than low thresholds, a statistically significant decline of ovarian reserve for the unexplained infertility group was found in the present study. This might be an undiagnosed reason for unexplained infertility.

\section{Conflict of interest}

The authors declare no conflict of interest.

\footnotetext{
References

1. Crosignani PG, Collins J, Cooke ID et al. Recommendations of the ESHRE workshop on 'Unexplained Infertility'. Anacapri, August 28-9, 1992. Hum Reprod 1993; 8: 977-80.
} 
2. Marc A, Fritz LS. Female infertility. In: Clinical Gynecologic Endocrinology and Infertility. $8^{\text {th }}$ ed. Speroff L, Fritz MA (eds.). Lippincott Williams \& Wilkins 2011; 1131-90.

3. Oner G, Ulug P, Elmali F. Ovarian reserve markers in unexplained infertility patients treated with clomiphene citrate during intrauterine insemination. Arch Med Sci 2015; 11: 1250-4.

4. van Rooij IA, Broekmans FJ, Hunault CC, et al. Use of ovarian reserve tests for the prediction of ongoing pregnancy in couples with unexplained or mild male infertility. Reprod Biomed Online 2006; 12: 182-90.

5. Murto T, Bjuresten K, Landgren BM, Stavreus-Evers A. Predictive value of hormonal parameters for live birth in women with unexplained infertility and male infertility. Reprod Biol Endocrinol 2013; 11: 61.

6. Leach RE, Moghissi KS, Randolph JF, et al. Intensive hormone monitoring in women with unexplained infertility: evidence for subtle abnormalities suggestive of diminished ovarian reserve. Fertil Steril 1997; 68: 413-20.

7. Practice Committee of American Society for Reproductive M. Diagnostic evaluation of the infertile female: a committee opinion. Fertil Steril 2012; 98: 302-7.

8. Guzick DS, Sullivan MW, Adamson GD, et al. Efficacy of treatment for unexplained infertility. Fertil Steril 1998; 70: 207-13

9. Scott RT Jr, Elkind-Hirsch KE, Styne-Gross A, et al. The predictive value for in vitro fertility delivery rates is greatly impacted by the method used to select the threshold between normal and elevated basal follicle-stimulating hormone. Fertil Steril 2008; 89: 868-78.

10. Frattarelli JL, Lauria-Costab DF, Miller BT, et al. Basal antral follicle number and mean ovarian diameter predict cycle cancellation and ovarian responsiveness in assisted reproductive technology cycles. Fertil Steril 2000; 74: 512-7.

11. La Marca A, Malmusi S, Giulini S, et al. Anti-Mullerian hormone plasma levels in spontaneous menstrual cycle and during treatment with FSH to induce ovulation. Hum Reprod 2004; 19: 2738-41.

12. Tsepelidis S, Devreker F, Demeestere I, et al. Stable serum levels of anti-Mullerian hormone during the menstrual cycle: a prospective study in normo-ovulatory women. Hum Reprod 2007; 22: 1837-40. 